\title{
DIGESTIBILIDAD IN SITU DE LA MATERIA SECA DE TRES DIETAS PARA OVINOS DE ENGORDA ${ }^{1}$
}

\author{
Jaime Salinas-Chavira², Juan Carlos Gutiérrez-González², Ramón García-Castillo , Ramiro López-Trujillo , \\ Arturo Duarte-Ortuño ${ }^{2}$
}

\section{RESUMEN}

Digestibilidad in situ de la materia seca de tres dietas para ovinos de engorda. El objetivo de este trabajo fue medir la digestibilidad in situ de materia seca (DISMS) de tres dietas de engorda para ovinos en Tamaulipas, México. Se usó la técnica de la bolsa de nylon para determinar la digestibilidad in situ y degradabilidad ruminal de la materia seca con tres borregos fistulados en el rumen y distribuidos en un diseño de Cuadro Latino. Los periodos de incubación ruminal fueron $0,4,8,12,24$ y 48 h. Todas los tratamientos $(\mathrm{T})$ incluyeron sorgo (Sorghum vulgare) molido como grano. El T1 y T2 se formularon con pulido de arroz (Oryza sativa) con 7,53\% en base seca (BS) y con $5 \%$ (BS) de soca de sorgo como forraje; respectivamente; y el T3 con $5 \%$ (BS) de zacate buffel (Cenchrus ciliaris) más 3\% (BS) de alfalfa (Medicago sativa). En T1 se observó el mayor valor $(\mathrm{P}<0,05)$ de digestibilidad a las 0 y $4 \mathrm{~h}$. Para las $24 \mathrm{~h}$, la mayor $(\mathrm{P}>0,05)$ fracción digestible fue para T2. A las 48 $\mathrm{h}$ no se observó diferencia entre tratamientos $(\mathrm{P}>0,05)$. La fracción rápidamente soluble y la constante de degradación fueron mayores $(\mathrm{P}<0,05)$ en $\mathrm{T} 1$ que T2 y T3. La dieta T1 presentó la fracción más digestible en las primeras horas de incubación en rumen $(0$ y 4). La dieta T3 se digirió en menor proporción; sin embargo, pudiera ser la mejor para la engorda de ovinos, por menor riesgo de acidosis.

Palabras claves: Digestión ruminal, raciones, borregos.

\begin{abstract}
In situ dry matter digestibility of three fattening diets for lambs. The objective of this work was the measure the in situ and ruminal degradability and dry matter digestibility of three fattening diets for lambs, used by lamb producers in the central area of Tamaulipas, México. The nylon bag technique was used to determine in situ dry matter digestibility, using three lambs with cannulas in the rumen, distributed in a Latin Square design. The ruminal incubation times were $0,4,8,12,24$ and $48 \mathrm{~h}$. All treatment diets (T) used ground sorghum grain (Sorghum vulgare) as a base. $\mathrm{T} 1$ included rice (Oryza sativa) polishing with $7.53 \%$ on a dry matter basis (DM) of sorghum straw as forage; T2 had $5 \% \mathrm{DM}$ of sorghum straw as forage; and T3 had 5\% DM of buffel grass (Cenchrus ciliaris) hay plus 3\% DM of alfalfa hay (Medicago sativa). In $\mathrm{T} 1$, the highest $(\mathrm{P}<0.05)$ value of digestibility at 0 and $4 \mathrm{~h}$ was observed. For $24 \mathrm{~h}$, the highest $(\mathrm{P}<0.50)$ digestible fraction was obtained with $\mathrm{T} 2$. At $48 \mathrm{~h}$, no difference $(\mathrm{P}>0.05)$ was observed between treatments. The washing loss and the degradation constant were higher $(\mathrm{P}<0.05)$ for $\mathrm{T} 1$ than $\mathrm{T} 2$ and $\mathrm{T} 3$. The diet of $\mathrm{T} 1$, containing rice polishing, produced the highest digestible fraction at the first hours of incubation in rumen $(0$ and $4 \mathrm{~h})$. T3 was digested in smaller proportion, although it could be the best diet for feedlots of lambs because of the lower acidosis risk.
\end{abstract}

Key words: Ruminal digestion, rations, sheep.

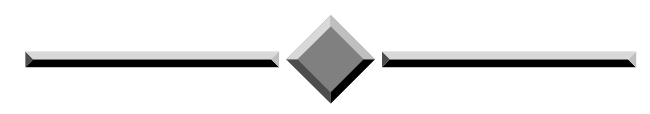

1 Recibido: 17 de junio, 2011. Aceptado: 3 de octubre, 2011. Presentado como tesis profesional por Juan Carlos Gutiérrez González.

2 Facultad de Medicina Veterinaria y Zootecnia, Universidad Autónoma de Tamaulipas, 5.5 km. carretera Victoria - Mante, Cd. Victoria 87000 Tamaulipas, México. Apartado Postal 263.jsalinasc@hotmail.com; aduarte@uat.edu.mx

3 Universidad Autónoma Agraria “Antonio Narro", Calzada Antonio Narro 1923, colonia Buenavista, Saltillo Coahuila, México. Código Postal 25315.rgarcas2009@hotmail.com; rlopez@uaaan.mx 


\section{INTRODUCCIÓN}

En México se está incrementando la engorda de ovinos con raciones altas en concentrados, existiendo para este fin alimentos comerciales, así como alimentos que elaboran los productores utilizando ingredientes que se encuentran disponibles en cada región del país. El pulido de arroz (Oryza sativa), está disponible en algunas regiones como Tamaulipas, en el noreste de México; y es utilizado por su aporte tanto de energía como de proteína, en la alimentación de ovinos. El pulido de arroz tiene alta degradabilidad ruminal (Rosales et al. 2005); por lo que, altos niveles en la dieta de ovinos en engorda pueden afectar el comportamiento productivo (Pérez et al. 2006). El alto aporte de energía del pulido de arroz se debe a su alto contenido de lípidos; no obstante, el comportamiento en engorda o características de la canal de ovinos no se afectan por el nivel de pulido de arroz junto con cebo de res en la ración (Salinas et al. 2008).

En el año 2007, se produjeron en México 6202920 t de sorgo en grano; de las cuales Tamaulipas (principal productor) genera 2490194 toneladas. Este grano forma la mayor proporción (60 a 75\%) de las raciones de engorda para ovinos. Como fuente de forraje se incluye la soca de sorgo (residuo de la cosecha del grano); aunque en otras ocasiones se usa heno de zacate buffel (Cenchrus ciliaris). Estos forrajes parcialmente se podrían substituir por un forraje de mayor contenido y digestibilidad de nutrientes como el heno de alfalfa (Medicago sativa). Existen indicios que pueden mejorar las raciones, por ejemplo Ramírez-Sánchez et al. (2008) utilizaron heno de alfalfa y soca de sorgo; concluyen que un nivel de forraje de $7 \%$ a $14 \%$ en la ración de engorda de ovinos produce similares ganancias de peso y eficiencia alimenticia. San Juan et al. (2008) encontraron que 3\% de heno de alfalfa mejora la ganancia de peso en la primera etapa de la engorda de los ovinos, aunque en la última etapa hay resultados similares a los encontrados con soca de sorgo.

En Tamaulipas, México, existen 240972 cabezas (2,9\% del total del país), con lo cual se ubica en los primeros diez estados más productores (SIAP 2010). Contando con sistemas de producción ovina de engorda en corral cuyas raciones es necesario evaluar para mejorar el desempeño productivo en la engorda de los ovinos. La evaluación de alimentos en la engorda intensiva de ovinos realizada por Mendoza et al. (2007) muestra que con la digestibilidad in situ de la materia seca (DISMS) y almidón se puede predecir el comportamiento en engorda de ovinos, y que puede influir en las utilidades económicas que recibe el productor. Esto es importante para la formulación de raciones, con la finalidad de obtener un incremento en la respuesta del ovino en engorda intensiva y así mejorar las utilidades del productor, incrementar la producción de carne de ovino en el país y reducir las importaciones. El objetivo de este trabajo fue medir la digestibilidad in situ de materia seca (DISMS) de tres dietas de engorda para ovinos.

\section{MATERIALES Y METODOS}

\section{Área de estudio}

El presente estudio se llevó a cabo de Abril a Septiembre de 2004 en la Facultad de Medicina Veterinaria y Zootecnia, Universidad Autónoma de Tamaulipas, en Ciudad Victoria, Tamaulipas (zona subtropical, noreste de México). El área está situada a $23^{\circ} 44^{\prime}$ ' $06^{\prime}$ " $\mathrm{N}$ y $97^{\circ} 09$ ' 50 ” O, a una altitud de 340 m. La precipitación media anual es de $900 \mathrm{~mm}$ y la temperatura promedio es de $25^{\circ} \mathrm{C}$ (INEGI 2006). Estas características son típicas de trópico seco.

\section{Dietas experimentales}

Se evaluaron tres dietas empleadas en la engorda de ovinos por productores en la región centro del estado de Tamaulipas (Cuadro 1). La característica principal de la dieta 1 (T1) es la inclusión de pulido de arroz con grano de sorgo en una proporción de concentrado y forraje de $92,47 \%$ y $7,53 \%$, en base seca (BS). La dieta 2 (T2) sin pulido de arroz incluyó 71\% de grano de sorgo; con una proporción concentrado:forraje de 95:5 en base seca. La dieta 3 (T3) incluyó 65,5\% sorgo grano $20 \%$ soya, y la proporción concentrado:forraje de 92:8 en base seca.

\section{Digestibilidad in situ de la materia seca}

Se utilizaron tres borregos de raza Pelibuey adultos (25 kg de peso inicial) no castrados, equipados con cánulas permanentes de rumen (40 mm de diámetro) y distribuidos en un diseño cuadrado latino de $3 \mathrm{x}$ 3 para determinar la DISMS. Antes del ensayo de 
Cuadro 1. Dietas integrales usadas por ovinocultores en la región centro de Tamaulipas (\% base seca). México, 2004.

\begin{tabular}{lccc}
\hline Ingredientes & Dieta $\mathbf{1}$ & Dieta 2 & Dieta 3 \\
\hline Sorgo, grano molido & 61,73 & 71 & 65,5 \\
Pulido arroz & 12 & 0 & 0 \\
Soca de sorgo & 7,53 & 5 & 0 \\
Melaza, caña & 7 & 6 & 4 \\
Pasta de soya & 6,86 & 15 & 20 \\
Premezcla mineral & 3 & 3 & 2,5 \\
Carbonato de calcio & 1 & 0 & 0 \\
Sulfato amonio & 0,42 & 0 & 0 \\
Sal, grano & 0,46 & 0 & 0 \\
Alfalfa, heno & 0 & 0 & 3 \\
Zacate buffel, heno & 0 & 0 & 5 \\
Nutrientes, base seca ${ }^{1}$ & & & \\
Proteína cruda, $\%$ & 11,02 & 13,82 & 16,28 \\
Energía metabolizable, Mcal/kg & 2,78 & 2,96 & 2,96 \\
Fibra detergente neutra, $\%$ & 20,99 & 18,28 & 19,85 \\
Fibra detergente neutra del forraje, $\%$ & 4,89 & 3,25 & 5,06 \\
\hline
\end{tabular}

${ }^{1}$ Estimado en base a las tablas de la NRC (2007).

digestibilidad los ovinos fueron tratados contra parásitos internos y externos e inyectados con una preparación de vitaminas A, D y E. Los corderos fueron alojados en jaulas de metabolismo individuales y se alimentaron ad libitum cada uno con las tres dietas (Cuadro 1) en dos comidas iguales a las 9:00 y las 16:00 h. El agua estuvo disponible permanentemente durante todo el experimento. Se utilizaron tres periodos experimentales, a cada uno se le asignó un período de adaptación de diez días antes de la incubación de las bolsas en el rumen.

En la incubación ruminal se utilizaron bolsas de nylon de $5 \times 10 \mathrm{~cm}$ y malla de $53 \mu \mathrm{m}$. Las bolsas fueron incubadas por duplicado, cada una con $6 \mathrm{~g}$ de la dieta que se ofreció a los corderos en cada periodo experimental. Las muestras de alimentos fueron molidas en un molino $\mathrm{N}^{\circ} 4$ equipado con una criba de retención de 2,0 mm. Las bolsas con la muestra de alimento estuvieron atadas a una cuerda de nylon de $20 \mathrm{~cm}$ con un peso de metal al final para garantizar que las muestras estuvieran inmersas en el saco ventral del rumen. Las bolsas fueron incubadas 4, 8, 12, 24 y
48 h. La degradación de materia seca a tiempo cero se estimaron con la ecuación de Ørskov y McDonald (1979). Después de cada incubación las bolsas fueron retiradas del rumen, se lavaron con agua a baja presión hasta que salió agua clara de la bolsa y luego se secaron en un horno de aire forzado a $60{ }^{\circ} \mathrm{C}$ durante $48 \mathrm{~h}$ a un peso constante. Las pérdidas de MS se estimaron por el cambio en peso de la muestra de alimento en las bolsas antes y después de la incubación ruminal, de acuerdo con la metodología de Ørskov y McDonald (1979), de la siguiente forma:

MS desaparecida $(\%)=[($ MS inicial-MS final $) /$ MS inicial] x 100

Los parámetros de degradación ruminal de la materia seca se estimaron con el modelo de Ørskov y McDonald (1979), modificado por McDonald (1981):

$\mathrm{P}=\mathrm{a}+\mathrm{b}(1-\mathrm{e}-\mathrm{c} * \mathrm{t})$

Donde: 
$\mathrm{P}=$ Degradación de la materia seca $(\%)$

$\mathrm{a}=$ Fracción rápidamente soluble

$\mathrm{b}=$ Fracción degradable

$\mathrm{a}+\mathrm{b}=$ Degradabilidad potencial $(\%)$

$\mathrm{c}=$ Tasa fraccional de degradación (h-1)

$\mathrm{t}=$ Tiempo $(\mathrm{h})$

Para estimar la degradabilidad efectiva de materia seca (DEMS) se usaron las constantes de flujo ruminal (k) de 1, 5, y 10\% h-1 (Ørskov and McDonald 1979): DEMS $=\mathrm{a}+\left(\mathrm{b}^{*} \mathrm{c}\right) /(\mathrm{c}+\mathrm{k})$

\section{Procedimientos estadísticos}

Los datos de la DISMS para cada hora de incubación así como los parámetros de degradación ruminal de materia seca se analizaron en un diseño en Cuadro Latino 3 x 3 con tres tratamientos, tres periodos experimentales y tres animales; con dos bolsas por incubación, para analizar un cuadro latino replicado. La comparación de medias de tratamientos se realizó mediante el procedimiento GLM (SAS 1998).

\section{RESULTADOS Y DISCUSIÓN}

En las 0 y 4 h de incubación en el rumen se observó menor digestibilidad de la materia seca $(\mathrm{P}<0,05)$ para el tratamiento T3, seguido por T2 y T1 (Cuadro 2). A las 8, 12 y 48 h de incubación, no se observó efecto de tratamiento sobre la digestibilidad in situ $(\mathrm{P}>0,05)$. En forma consistente, se observó mayor $(\mathrm{P}<0,05)$ degradabilidad de la fracción soluble en el T1 (Cuadro 3). La rápida digestibilidad ruminal de la materia seca en rumen de la dieta 1 se debe a que contiene más altos niveles de melaza y pulido de arroz. En este sentido Maynard et al. (1983) y Linares et al. (2003) mencionan que el almidón, el azúcar de caña y las melazas son de fácil digestión para los microorganismos presentes en el rumen. Fortanelli (1996) reportó en ovinos mayor degradabilidad ruminal con ensilaje de punta de caña de azúcar (ensilaje de cogollo de caña de azúcar) con urea y melaza en relación al control. Rosales et al. (2005) concluyeron que la dieta control sin pulido de arroz se degrada más lento en rumen, aunque la degradabilidad ruminal total es similar entre las dietas estudiadas. Esto concuerda con la presente investigación, ya que el pulido de arroz presenta mayor tasa de digestión ruminal que el grano de sorgo.

Otro factor que pudo haber contribuido es que el grano de sorgo presenta la menor tasa de digestión en rumen (Herrera- Saldaña et al. 1990) por lo que la ración de $\mathrm{T} 2$, con mayor cantidad de grano de sorgo presentó menores digestibilidades a las 0 y 4 h que T1. Comparado T2 y T3, esta última presentó menor tasa de digestión ruminal a las 0 y 4 h. Esto se puede explicar por la diferencia en la proporción de forraje y concentrado. T3 tiene mayor proporción de forraje que T2 y por tanto menor digestibilidad ruminal de la materia seca. Papi et al. (2011) en corderos

Cuadro 2. Digestibilidad in situ de la materia seca de las dietas (\%) para engorde de ovinos en la región centro del estado de Tamaulipas. Tamaulipas, México, 2004.

\begin{tabular}{cccccccc}
\hline $\begin{array}{c}\text { Tiempo de incubación } \\
\text { (h) }\end{array}$ & \multicolumn{3}{c}{ Dieta } & \multicolumn{3}{c}{ Contrastes (Probabilidad) } & EE \\
\hline 0 & T1 & T2 & T3 & T1 vs T2 & T1 vs T3 & T2 vs T3 \\
\hline 4 & 44,62 & 38,77 & 33,88 & $<0,01$ & $<0,01$ & 0,02 & 1,12 \\
8 & 48,06 & 45,89 & 43,11 & 0,02 & $<0,01$ & 0,01 & 0,51 \\
12 & 55,04 & 54,65 & 53,05 & 0,77 & 0,16 & 0,25 & 0,91 \\
24 & 55,96 & 56,04 & 56,96 & 0,95 & 0,51 & 0,55 & 1,64 \\
48 & 64,89 & 71,40 & 64,34 & 0,02 & 0,82 & 0,02 & 1,64 \\
\hline
\end{tabular}

T1 (61,7\% grano de sorgo, $12 \%$ pulido de arroz, $7,53 \%$ soca de sorgo; T2 (71\% sorgo grano, $5 \%$ soca de sorgo); T3 $(65,5 \%$ grano de sorgo, $3 \%$ alfalfa, $5 \%$ zacate buffel).

$\mathrm{EE}=$ Error estándar de la media. 
Cuadro 3. Parámetros de degradabilidad ruminall de las dietas experimentales cuando se incubaron en el rumen de los ovinos. Tamaulipas, México, 2004.

\begin{tabular}{lccccccc}
\hline & \multicolumn{3}{c}{ Dieta } & \multicolumn{5}{c}{ Contrastes (Probabilidad) } \\
\cline { 2 - 6 } Tiempo de incubación, $\mathbf{~}$ & T1 & T2 & T3 & T1 vs T2 & T1 vs T3 & T2 vs T3 & EE \\
\hline Rápidamente soluble, a & 43,58 & 38,12 & 37,16 & $<0,01$ & $<0,01$ & 0,43 & 0,83 \\
Degradabilidad efectiva, b & 49,77 & 54,82 & 53,23 & 0,15 & 0,31 & 0,63 & 2,28 \\
Degradabilidad potencial, a+b & 93,36 & 91,80 & 90,37 & 0,87 & 0,37 & 0,45 & 2,21 \\
Constante de degradación, c & 0,025 & 0,04 & 0,04 & $<0,01$ & $<0,01$ & 0,80 & 0,003 \\
\hline Degradabilidad efectiva estimada a tasa de paso $\left(\mathrm{h}^{-1}\right)$ de: & & & & & \\
\hline 0,01 & 78,90 & 81,58 & 78,92 & 0,23 & 0,99 & 0,23 & 1,47 \\
0,05 & 60,08 & 62,20 & 60,75 & 0,10 & 0,57 & 0,23 & 0,79 \\
0,10 & 53,48 & 53,63 & 52,63 & 0,84 & 0,27 & 0,20 & 0,50 \\
\hline
\end{tabular}

${ }^{1}$ Estimados con la ecuación de Orskov y McDonald (1979).

T1 (61,7\% grano de sorgo, $12 \%$ pulido de arroz, 7,53\% soca de sorgo; T2 (71\% sorgo grano, 5\% soca de sorgo); T3 (65,5\% grano de sorgo, $3 \%$ alfalfa, $5 \%$ zacate buffel).

$\mathrm{EE}=$ Error estándar de la media.

usaron diferentes proporciones de concentrado y de forraje como heno de alfalfa (BS) de 70:30 (C30), 50:50 (50), 30:70 (C70), 10:90 (C90). Encontraron una reducción lineal $(\mathrm{P}<0,05)$ en consumo de BS y conversión alimenticia y cuadrática para la ganancia de peso, además de incremento lineal $(\mathrm{P}<0,05)$ en el rendimiento en canal, y sin efecto en las características de la canal $(\mathrm{P}>0,05)$ por efecto del incremento de concentrado en la dieta. De acuerdo con estos resultados, Tripathi et al. (2007) también encontraron incremento en la producción de ovinos al incrementar el nivel de concentrado en la dieta. Por otra parte, las raciones de engorda de corderos utilizan niveles de concentrado en la dieta cercanos a $90 \%$, esto con el objetivo de máximo consumo de energía, sin embargo en estos altos niveles de concentrado no siempre se encuentra mejora en el comportamiento productivo de los ovinos en engorda. Además, no se ha encontrado efecto en el comportamiento en engorda de ovinos con niveles de concentrado de 86 a 93\% (Ramírez-Sánchez et al. 2008).

Es de notar que $\mathrm{T} 1$ con similar proporción de forraje y concentrado que T3, presentó mayor tasa de DISMS; lo que se puede atribuir a la inclusión de pulido de arroz y melaza. Una respuesta similar al presente estudio en cuanto a la degradabilidad ruminal de raciones con melaza, grano de sorgo y salvado de trigo fue obtenida por Linares et al. (2003). También se ha encontrado mayor digestibilidad de la materia seca en las dietas con mayores niveles de melaza (Romero et al. 1985). Así mismo, mayor digestibilidad de raciones con urea (Medina et al. 1995, Hernández 1991). Lo anterior explica la mayor la digestibilidad de T2 en relación a T3, ya que T2 contenía mayor proporción de melaza que es un ingrediente de rápida absorción, por lo tanto mayor digestibilidad que el tratamiento T3.

Para las $24 \mathrm{~h}$ de incubación, la mayor digestibilidad fue para $\mathrm{T} 2$, seguida por T1 y T3 $(\mathrm{P}<0,05)$. Las DISMS fueron para este periodo de incubación de 64,$89 ; 71,39$ y $64,34 \%$ respectivamente para T1, T2 y T3. En la evaluación de alimentos integrales para la engorda intensiva de ovinos, reportan DISMS a $24 \mathrm{~h}$ de incubación que van de 62,10 a 76,12\% (Mendoza et al. 2007). Estas digestibilidades son similares a las del presente trabajo. También se reporta que la mayor digestibilidad in situ a 24 h correspondió a la ración con mayor contenido de almidón (grano) (Mendoza et al. 2007). En la presente investigación la mayor digestibilidad in situ también correspondió a la dieta con mayor contenido de grano de sorgo. En el mismo sentido Rojo-Rubio et al. (2007) mencionan que la tasa y extensión de la degradación ruminal del almidón está determinada por la relación intrínseca de factores alimentarios como fuente de almidón, composición 
química y nutritiva de la dieta, la cantidad de alimento consumida por unidad de tiempo, procesamiento de granos y adaptación de los microorganismos ruminales a la dieta.

Para las 48 h de incubación no se encontró efecto de tratamiento sobre la digestibilidad in situ de las dietas $(\mathrm{P}>0,05)$. La alta digestibilidad de las dietas, en las primeras horas de incubación, se puede atribuir a su alto contenido de concentrados. En raciones para ovinos de engorda altas en concentrado y con diferentes niveles de mazorca de maíz completa (incluye grano, hoja y olote), se reportó que la degradabilidad efectiva de la materia seca no se afectó en velocidades de paso de medio a alto (Salinas-Chavira et al. 2011), lo cual también es esperado para las dietas del presente estudio.

Los resultados del presente estudio también se pueden explicar por las características físicas y químicas de la fibra, y principalmente por la fibra detergente neutra (FDN). La dieta del T1 observó la mayor tasa de digestión en las primeras horas de incubación ruminal; esta dieta contiene el más alto porcentaje de FDN, sin embargo el pulido de arroz aporta cerca de $4 \%$ de FDN, pero el pequeño tamaño de partícula del pulido de arroz (polvo) hacen que su velocidad de paso sea muy rápida, a pesar de su alto nivel de FDN. De acuerdo con esto Parish y Rhinehart (2008) señalan que la fibra finamente molida pasa rápidamente por el sistema digestivo y no llena los requerimientos de fibra efectiva del ganado. El T2 presentó resultados intermedios en la tasa de digestión ruminal en las primeras horas de incubación. Esta dieta contiene los menores porcentajes de FDN, a pesar de eso se digiere más lento que la dieta $\mathrm{T} 1$, posiblemente relacionado a lo mencionado anteriormente del pulido de arroz. La dieta del T3 en este estudio mostró la menor tasa de desaparición de la materia seca en las primeras horas de incubación ruminal. Esta dieta contiene un nivel intermedio de FDN $(19,85 \%)$, pero es la dieta que tiene mayor cantidad de FDN del Forraje $(5,06 \%)$. Esto contribuye a explicar la menor tasa de digestión de la materia seca de esta dieta. En este estudio los porcentajes de FDN proveniente del forraje estimados fueron 4,$89 ; 3,25$ y $5,06 \%$, respectivamente para T1 a T3. A esta fracción de forraje se le conoce como forraje funcional, y es aquella que contribuye a estimular la masticación, la rumia y la motilidad ruminal. En la formulación de raciones para ganado de engorda se estima que el porcentaje de FDN funcional (del forraje) debe ser de 6 a 9\% para máxima respuesta productiva (Zinn y Ware 2007). Aunque no se encontraron estudios similares en ovinos, al parecer las tres dietas estudiadas se podrían beneficiar con pequeñas cantidades de forraje adicional en la dieta.

En la engorda intensiva de ovinos se han reportado diferencias en consumo y ganancia de peso con diferentes alimentos integrales (Mendoza et al. 2007) debido tanto a la concentración de nutrientes como al uso de distintos aditivos que pueden influir en el metabolismo, digestibilidad y posiblemente en el control de acidosis subaguda. En el presente estudio se usaron dietas altas en concentrado, y T1 de muy rápida digestión; en ninguna de las tres dietas se usaron aditivos. El bicarbonato de sodio, las levaduras (probioticos) y los ionóforos mejoraría el medioambiente ruminal, la digestión en general, la ganancia de peso y eficiencia de utilización de alimento de los ovinos en engorda.

La composición de las dietas influye sobre la DISMS. La dieta 1 (con pulido de arroz) incluyó los ingredientes de mayor digestibilidad ruminal de la materia seca en las primeras horas de incubación, seguida por la dieta 2 (5\% de forraje). La alta digestibilidad en las dietas 1 y 2 podría generar un $\mathrm{pH}$ ácido en rumen y afectar la producción de ovinos en engorda intensiva. Por lo tanto, en el presente estudio se concluye que la dieta 3 es la mejor, debido a que incluye $8 \%$ de forraje con lo que presenta un mejor balance de digestibilidad in situ de la materia seca, con menor riesgo de acidosis ruminal por la menor degradabilidad ruminal de la materia seca; y los ovinos con esta dieta podrían rendir mejor producción en la engorda intensiva.

\section{LITERATURA CITADA}

Fortanelli, HLM. 1996. Digestibilidad ruminal de materia seca en dietas integrales para ovinos con distintos ensilajes de caña adicionados con urea y melaza. Tesis Licenciatura. Facultad de Medicina Veterinaria y Zootecnia. Universidad Autónoma de Tamaulipas. Cd. Victoria, Tamaulipas, México. 43 p.

Hernández, BJD. 1991. Fisiología digestiva de los rumiantes manipulados del rumen con dietas a base de paja. Universidad Autónoma de Chihuahua. Tesis Doctorado. Chihuahua, México. 136 p.

Herrera-Saldaña, R; Huber, JT; Poore, MH. 1990. Dry matter, crude protein and starch degradability of five cereal grains. J. Dairy Sci. 73:2386-2393. 
INEGI (Instituto Nacional de Estadística, Geografía e Informática). 2006. Sistema para la Consulta del Anuario Estadístico del Estado de Tamaulipas. Acceso, 16 junio de 2011. Disponible en http://www.inegi. org.mx/est/contenidos/espanol/sistemas/aee06/estatal/ tam/index.htm

Linares, CP; Salinas, CHJ; Hernandez, BD. 2003. Digestibilidad in situ y degradabilidad ruminal de raciones con distintos niveles de lípidos de baja biohidrogenación ruminal. XXXI Reunión Anual de la AMPA, Phoenix, Arizona, EUA. p. 193-198.

Maynard, LA; Loosli, JK; Hintz, HF; Warner, RG. 1983. Nutrición Animal, MC GRAW-HILL. México. p. 22-48.

McDonald, I. 1981. A revised model for the estimation of protein degradability in the rumen. Journal of Agriculture Science 96:251-252.

Medina, LJB; Salinas, CHJ; Lerma, DEC; Martínez, DR; Yado, PR. 1995. Digestibilidad in vivo del ensilaje de baya madura de calabaza (Cucurbita máxima) con soca de sorgo, urea y melaza en raciones integrales para ovinos. Memoria VII Congreso Nacional De Producción Ovina AMTEO Chapingo, México. p. 77-81.

Mendoza, MGD; Plata, PFX; Ramirez, MM; Mejía, DMA; Lee, RH; Bárcena GR. 2007. Evaluación de alimentos integrales para el engorde intensivo de ovinos. Revista Científica, FCV-LUZ. 1:66-72.

NRC. 2007. National Research Council. Nutrient requirements of small ruminants. Sheep, goats, cervids, and new world camelids. Washington, D.C.: The National Academy Press. 362 p.

Ørskov, ER; Mcdonald, I. 1979. The estimation of protein degradability in the rumen form incubation measurements weighted according to rate of passage. Journal of Agricultural Science (Cambridge) 92:499-503.

Papi, N; Mostafa-Tehrani, A; Amanlou, H; Memarian, M. 2011. Effects of dietary forage to concentrate ratios on performance and carcass characteristics of growing fat-tailed lambs. Animal Feed Science and Technology 163:93-98.

Parish, JA; Rhinehart, JD. 2008. Fiber in beef cattle diets. Mississippi State University Extension Service. Publication 2489. p 1-7.

Pérez, HJA; Salinas, CHJ; García, CRF; Arzola, AAC. 2006. Efecto de raciones con distintos niveles de pulido de arroz sobre el comportamiento productivo de ovinos en engorda. Memorias de la XXXIV Reunión Nacional de la Asociación Mexicana de Producción Animal. p. $80-83$.
Ramírez-Sánchez, R; Salinas-Chavira, J; Guevara-Guerrero, M. 2008 Efecto del nivel de forraje y concentrado en raciones sobre el comportamiento productivo de ovinos en engorda. Memorias de XIV Congreso Nacional de la Asociación Mexicana de Técnicos Especialistas en Ovinos. p. 1-5.

Rosales, VJA; Salinas, CHJ; Arzola, AC; Jiménez, CJ; Loredo, OJ; García, CRF. 2005. Efecto del nivel de pulido de arroz sobre la degradabilidad ruminal de materia seca de dietas de engorda para ovinos. Biotam. p. 273-275.

Rojo-Rubio, R; Mendoza-Martínez, GD; Montañez-Valdez, OD; Rebollar-Rebollar, S; Cardoso-Jiménez, D; Hernández-Martínez, J; González-Razo, FJ. 2007. Enzimas amilolíticas exógenas en la alimentación de rumiantes. Universidad y Ciencia Trópico Húmedo. 23:173-182

Romero, GH; Gómez, AR; Llamas, LG. 1985. Cambios en la digestibilidad y valor energético de raciones preparadas de melaza y grano de sorgo para finalizar novillos. Consultado 9 jun. 2011. Disponible en http://Patrocipes.uson.mx/Patrocipes/invpec/nutricion/N85011.htm

Salinas-Chavira, J; Guerrero, VI; Robles, CA; MontañoGómez, MF; Montañez-Valdez, OD. 2008. Effect of tallow and rice polishings in feedlot rations on growth and carcass characteristics of lambs. J. Appl. Anim: Res. 34:45-48.

Salinas-Chavira, J; Muñoz-de-Alba, M; Montañez-Valdez, OD; Martinez-Zapien, O. 2011. In vitro and in situ evaluation of feed lot rations with different levels of complete ear of the corn (Zea mays) in lambs. Indian Journal of Animal Sciences 81(3):289-92.

San Juan, MR; Salinas, CHJ; Yado, PR; Anaya, AD. 2008. Efecto de la calidad de forraje sobre el comportamiento productivo de ovinos en engorda. Memorias XXXII de Congreso Nacional de Buiatria. p. 462.

SAS. 1998. User's Guide: Statistics, SAS Inst. Inc. Cary. North Carolina. U. S. A. p. 558.

SIAP. 2010. Servicio de Información Agroalimentaria y Pesquera, México (en línea). Consultado 24 set. 2011. Disponible http://www.siap.gob.mx/

Tripathi, MK; Chaturvedi, OH; Karim, SA; Singh, VK; Sisodiya, SL. 2007. Effect of different levels of concentrate allowances on rumen fluid $\mathrm{pH}$, nutrient digestion, nitrogen retention and growth performance of weaned lambs. Small Ruminant Research 72:178-186.

Zinn, RA; Ware, RA. 2007. Forage quality: digestive limitations and their relationships to performance of beef and dairy cattle. 22 Annual Southwest Nutrition \& Management Conference. Tempe, AZ. p. 49-54. 